\title{
FAST FUNCTIONAL TEST GENERATION USING AN SDL MODEL
}

\author{
Robert L. Probert, Alan W. Williams \\ School of Information Technology and Engineering \\ University of Ottawa \\ Ottawa Ontario KIN 6N5, Canada \\ $\{$ bob,awilliam\}@site.uottawa.ca
}

\begin{abstract}
This paper reports the results of a successful study undertaken to determine the suitability of CASE tools and formal methods for systematic, rapid generation of functional test cases. In particular, the study involves the use of Message Sequence Charts (MSCs) [1], Specification and Description Language (SDL) [2] and Tree and Tabular Combined Notation (TTCN) [3] and the use of the Telelogic Tau tool set [4] which supports all three of these languages.
\end{abstract}

Keywords: functional testing, SDL, TTCN, MSC, formal test design, time to market

\section{INTRODUCTION}

The objective of the study was to generate functional tests for a telephone switching system. This was an unusual use of the TTCN test language, which has primarily been used for conformance testing. Recently, ITU has opened several new questions involving extensions to the definition, capabilities, and applicability of TTCN and Concurrent TTCN, including the ability to specify functional testing and performance testing. We see our work as contributing to this work on extending and improving upon the current versions of TTCN. 
A secondary, but equally important objective was to demonstrate the ability of our "fast to text/first to test" approach to dramatically compress the test cycle, and so to improve the time-to-market (TTM) of the corresponding product. The advantage of our approach is that it is no longer necessary to convince design engineers of the merits of a formal description technique. Instead, the test designers are able to use it at the beginning of the development process so that designers have high-yield scenarios in MSC form to use to validate their designs, often before any code is generated. This provides a cost-effective infrastructure for scenario-directed design. Others have proclaimed the benefits of scenario-directed design, but we may be the first to suggest a cost-effective approach acceptable to industry (no overhead on designer time). Here, we utilize the scenarios (those most likely to detest the presence of serious design errors or omissions).

A tertiary objective was to demonstrate to designers the value of using an FDT-based representation method supported by an industrial-strength (scalable) CASE tool set. Eventually, if designers see the cost-effectiveness of this more formal approach, they will adopt it. Testers already know its value.

Functional tests for a basic call, plus calls using four call processing features were derived. Two different models of phone sets were used: a basic set, and a set with an alphanumeric display screen and various lamps and feature keys. By employing the CASE tool coverage analysis features, coverage of all reachable transitions was ensured. Overall, we were able to generate high quality functional test cases in a reduced time interval.

As a result of the study, we have determined recommendations for enhancements to the languages and tools. We have also developed and applied some productivity measures that support the cost-effectiveness of this approach.

\subsection{The Project in Context}

In the industrial environment, it is unlikely that a fully complete and correct set of requirements will be determined before developing software. Usually, there is an initial set of requirements, and some development work is done using those requirements as a starting point. As a result of the first phase of development work, modifications are often made to the requirements. Aside from uncovering errors in the first version of the requirements, the consideration of exceptional cases may lead to new scenarios being developed. An initial prototype may lead to additional or modified requirements. 
After a first version of software is released, developers repeat the whole process for the next version of software, which undoubtedly will have additional features requested by customers. Recommendations for improving the software development process have to take into account that much of industrial software development is inserting new functionality into existing code.

As a result, we wanted replicate this iterative and incremental process in the study. Figure 1 shows the process that we want to follow. We are taking a use case-based approach, where the use cases are captured as Message Sequence Charts (MSCs). From an initial set of MSCs, we developed an executable SDL model. This allowed us to simulate and validate the model. Simulation allows us to execute the model interactively, and observe that it behaves as expected. Validation allows us to explore the state space of the model to detect properties such as deadlocks, and unspecified message receptions. Building the SDL model also produced new scenarios that were added to our set of MSC use cases. Once we were satisfied with the model, we could proceed to generate abstract TTCN test cases based on the use cases.

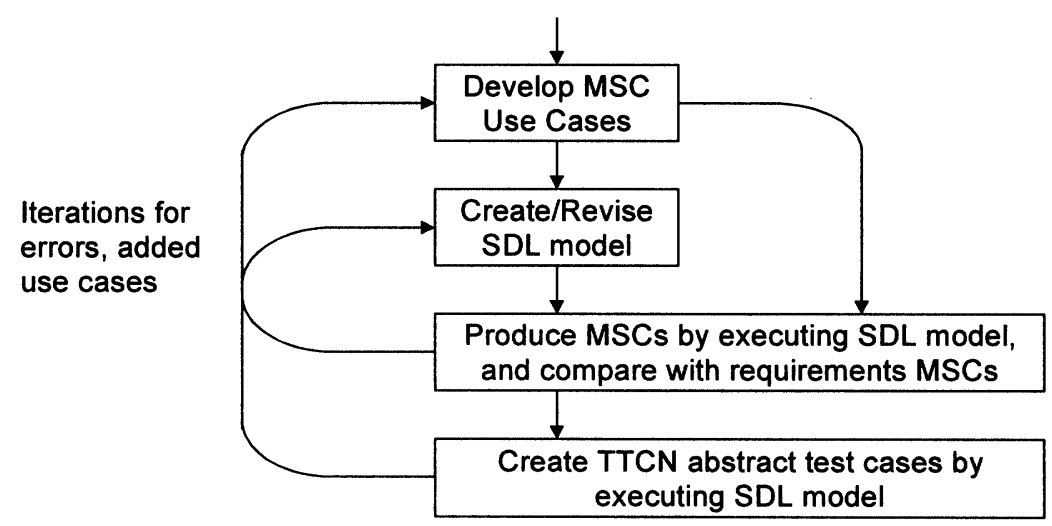

Figure 1. The Iterative, Incremental Development Process

\section{FUNCTIONAL VS. CONFORMANCE TESTING}

In this study, our objective was to generate functional tests rather than conformance tests. Conformance testing typically concentrates on the exchange of protocol messages both for their sequence and content. The goal is to verify system interoperability by conformance to a standard. 
The goal of functional testing is to take a user's view of the system, and check that customer requirements have been met. In particular, the actions and reactions of the system are considered from the user's viewpoint, to ensure that the system behaves as the user expects.

There is a distinction to be made between taking a "customer" viewpoint and an "end user" viewpoint in functional testing. While the direct customer of a telephone switch manufacturer is a company that offers telecommunications services, the end user is someone who makes phone calls. In our project, we took the "end user" view, and considered only the actions of the switch as could be controlled or observed from a phone. If we were taking a "customer" viewpoint, we also would have modelled and checked the behaviour of the operations and maintenance console for the switch.

These goals are reflected in the specific types of actions taken during testing, and reactions observed from the system under test. A functional test action might be to go offhook on a phone set. The corresponding conformance test action would be to construct and send a protocol message to the telephone switch as a result of going offhook. Similarly, a functional testing observation would be to check that the dial tone is heard, and various lamps and displays on the phone are updated. The conformance testing observation would be to check the exact sequence and data format of messages that are sent to the phone as a result of going offhook.

Functional testing also includes the detection of the presence and absence of persistent events. When taking a phone offhook, the dial tone should not only be started, but it should remain on until the user has dialled the first digit. Then, it should be turned off. We should be able to check that the dial tone is on at any time during the appropriate interval, and that it is off afterward.

Both types of testing are necessary. Functional testing is more indirect with respect to the system under test, in that we are using a phone to construct the protocol messages. With conformance testing, we would construct our own messages (correct, or not), and send them directly to the system under test. Conformance testing is to detect that the exchange of protocol messages is correct and robust. Functional testing is to confirm that the system behaves as according to customer or user expectations. 


\section{TEST MODEL ARCHITECTURE DEVELOPMENT}

In this section, we explain how we decided what to include in, and exclude from, our test model in SDL.

We decided to work backwards from our goal of running TTCN test cases in an automated execution environment, and seeing what constraints we had to satisfy in both the SDL model, and in how to format our MSC use cases that would drive the test execution.

\subsection{Mapping the MSC model to the test environment}

Because we are taking an end user view, functional testing of a telephone switch is necessarily an indirect process. In our study, we determined that the functionality of interest was that the interaction between a phone set and a switch exhibited the correct behaviour when using a phone. Therefore, our test actions will be to take the phone on or off hook, and press keys on the set. Our test observations will consist of observing the lamps, displays, and detecting the tones produced by the phone speaker in the handset.

Another element of indirection comes from the nature of telephone calls. If we are interested in observing that an incoming call works properly on our phone set of interest, another phone must be used to stimulate the switch to produce the incoming call notification. Figure 2 illustrates these situations.

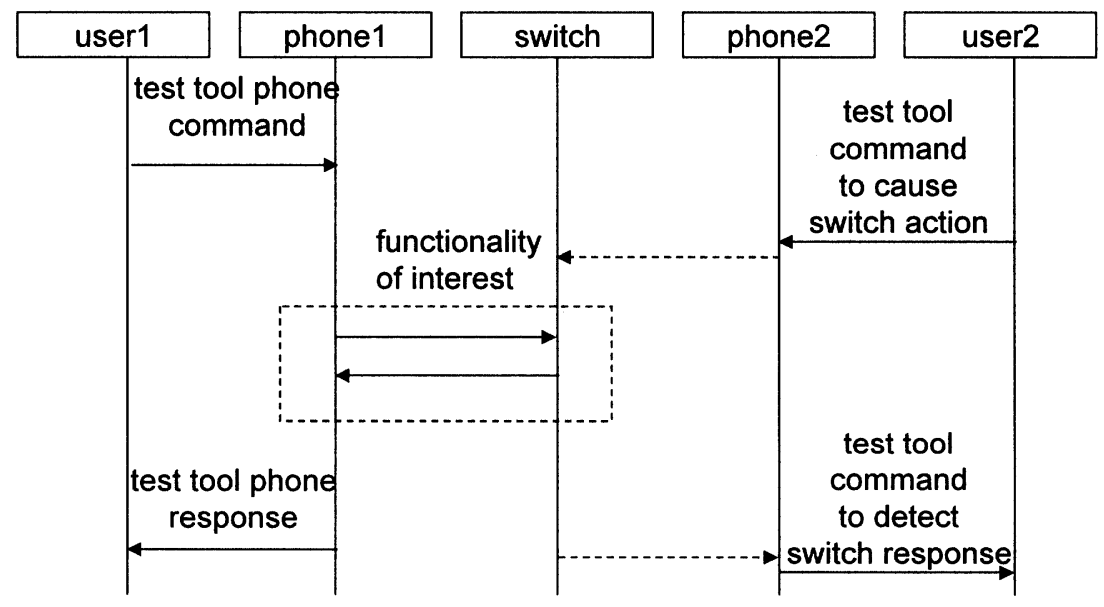

Figure 2. Mapping the MSC model to the test environment 
Eventually, what is described in Figure 2 as "test tool" commands or responses has to become TTCN send and receive commands to execute tests in the automated execution environment.

The particular environment, for which the test cases are targeted, has a TTCN compiler with an "adaption layer" that contains information about the specific protocol implemented in the system. That is, the adaption layer converts TTCN messages to specific commands that will drive the protocol interface to the system under test. Figure 3 illustrates this architecture.

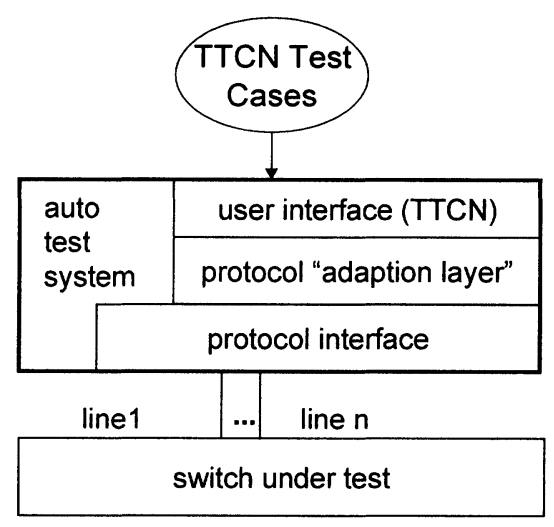

Figure 3. Test Architecture

The result, from the test case perspective, is that the adaption layer defines the legal set of TTCN messages that we are allowed to use. Therefore, our TTCN test cases must use those same message names and formats to work with the automated test execution environment. Since we are generating the TTCN tests automatically with a tool from an SDL model, this in turn means that the SDL model must also use these exact same message names and formats. Figure 4 shows this correspondence.
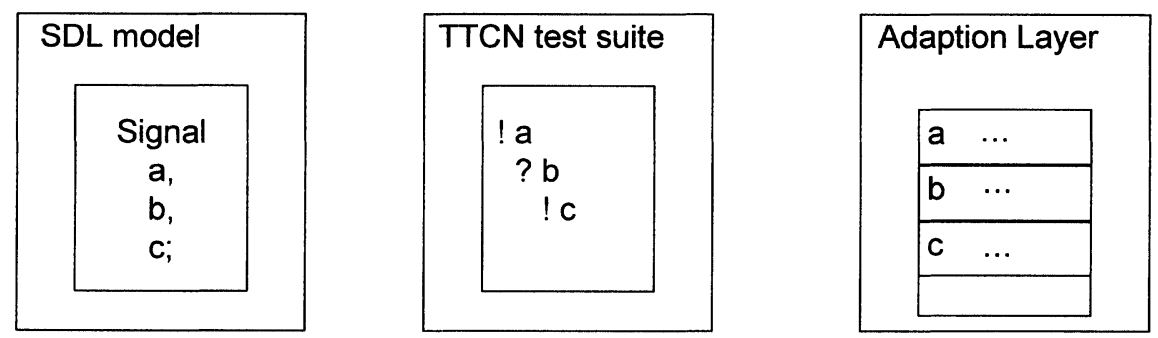

Figure 4. Determining SDL message names based on the Adaption layer 
This correspondence goes one step further when MSCs are used to drive the test generation tool to produce TTCN test cases automatically. The names of the messages in the MSCs must also correspond with those in the $\mathrm{SDL}$, at least at the boundary between the environment and the system. We shall discuss more about MSC creation in the next section.

\section{CREATING THE MSCS}

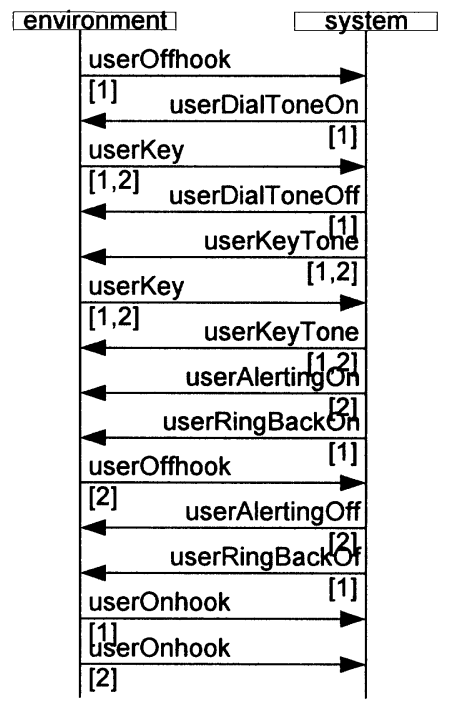

Figure 5. A system level message sequence chart

MSCs were used as our method for capturing use case scenarios for the project. MSCs are excellent for review purposes, as customers easily understand them. We used the MSCs to confirm that we had correctly captured scenarios. The first MSC we produced was for a basic normal call.

In the second stage of the project, we also captured four call processing features, to determine how difficult or easy it is to add functionality incrementally.

At this stage, we are not trying to impose architecture on the system. Therefore, the MSCs that are produced are "system level" MSCs in that they capture only the interactions between the system and the environment. For our functional testing viewpoint, this means capturing the interactions between a user and the various phones used in making phone calls. In particular, the automated execution environment would take on the role of all 
the phones, and therefore we are capturing the interactions between the test environment interface and the user. Figure 5 shows the type of MSC produced for a basic normal call.

Because the system level MSC exactly captures the commands that we need to send to the automated test execution environment, it can also be used as a basis to drive the automated execution of TTCN tests. Each message in the MSC in Figure 5 will be converted into a TTCN send or receive command by the test generation tool.

\section{CREATING THE SDL TEST MODELS}

In this study, we developed several iterations and versions of SDL test models. The first version was to produce a test model for telephone calls with no additional features.

While our original MSC scenario only describes a successful call, once this is captured in the model, we can start to ask "what if...?" questions to examine possible alternatives and exceptions. This is extremely important because it is likely that the software development team will implement normal scenarios correctly. It is the exceptional cases where errors are most likely to be found during testing phases. Therefore, to produce effective, high-yield tests, we must build exceptions into our test model. The stateoriented nature of SDL allows the model builder to systematically ensure that, for example, incoming calls have been accounted for in every single state.

Once we had created a model of telephone calls with no extra features, we developed two more versions of SDL test models. One was to determine how difficult or easy it was to add additional call-processing features. In essence, this is adding to the functionality of the telephone switch. Our second additional model was to investigate adding to the functionality of the phone set. That is, we added features such as a display screen, additional function keys, and multiple lamp indicators. This still impacts the functionality of the switch, as the switch has the responsibility of controlling the operation of these additional features on the phone set.

\section{$5.1 \quad$ Scope of the model}

For testing purposes, the SDL model is not intended to replicate the entire system behaviour of the switch and phone sets. Instead, it should reflect the goals of requirements capture, and of testing those requirements. It is possible that the models we produced could be used as inputs to the detailed 
design process for further refinements, and to include behaviour that is internal to the system.

For functional testing, the model should include:

1. the functionality to be tested

2. additional behaviour extending to points of control and observation, for test tool commands

3. enough internal behaviour to execute the model

The model does not need to include internal behaviour of the system that cannot be observed by test equipment, and is not part of the protocol.

\subsection{Simulated Execution of the SDL model}

By using the SDL tool simulated execution facility, the user can interact with the model, and view execution graphically. The user can record the execution as an MSC, and inspect the resulting MSC for anomalies.

The execution MSC can also be compared against the equivalent requirements MSC to see if it is consistent. This allows the model builder to confirm that the model correctly implements the initial requirements.

The requirements MSCs and the execution MSCs are not identical though. They should be consistent in the exchange of messages between the system and environment. But they can differ because of extra information that results in building the SDL model. The SDL model contains states and these are automatically inserted into the execution MSCs. The SDL model implemented timers, and the setting, expiry, and cancellation of timers also appear in the execution MSCs. Also, we found that what was originally envisaged as two original messages were consolidated into a single message with parameters. Finally, the execution MSCs also contains architectural information inside the system, including internal messages sent between system components. Figure 6 shows an example of an execution MSC that highlights differences from the original model. 


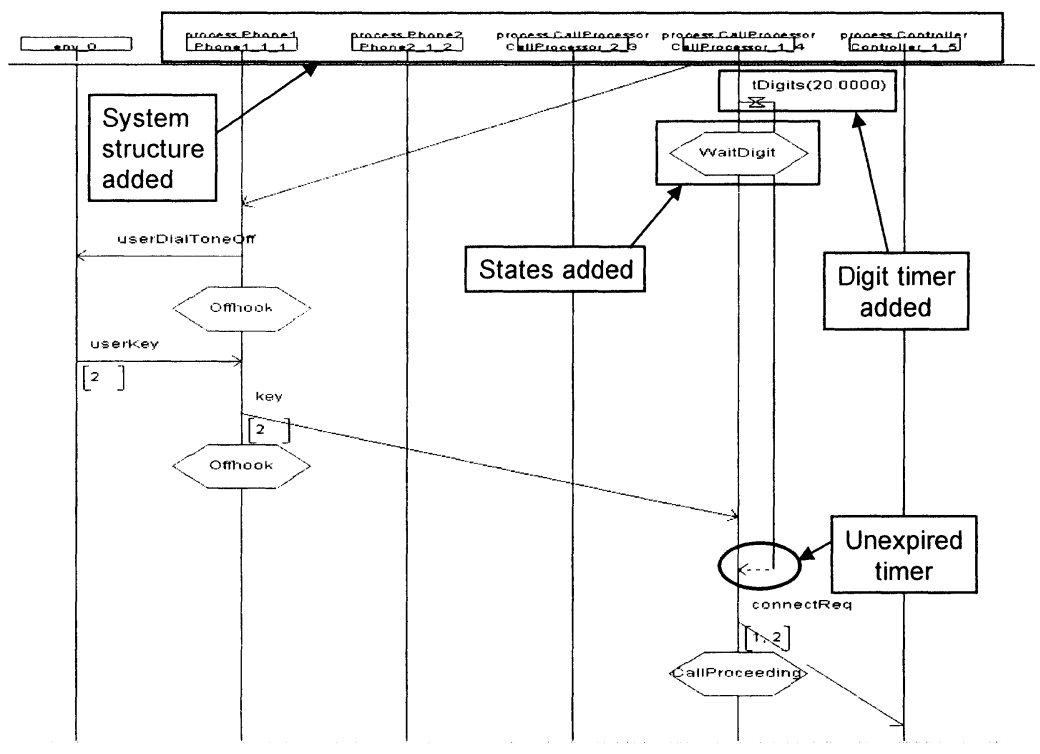

Figure 6. Differences in an execution MSC

\section{FINDING A BUG USING STATE SPACE EXPLORATION}

We also checked the SDL model using a state space validation tool. The validator checks for deadlocks, unspecified receptions, and can determine the consistency of an MSC with the SDL model.

The output from the tool for any anomalies consists of a description of the problem found, and an MSC scenario that describes what had happened up to the point when the problem was discovered.

In our study, we ran the validator on our SDL model, and found that an unspecified reception turned out to be a bug in our SDL model of the call back feature (illustrated in Figure 7). The feature is activated after a called party did not answer a call. We forgot to have the feature activation terminate the ringing of the called phone. The (still ringing) phone was later answered, but the "answer indication" reception was unspecified at the caller. 


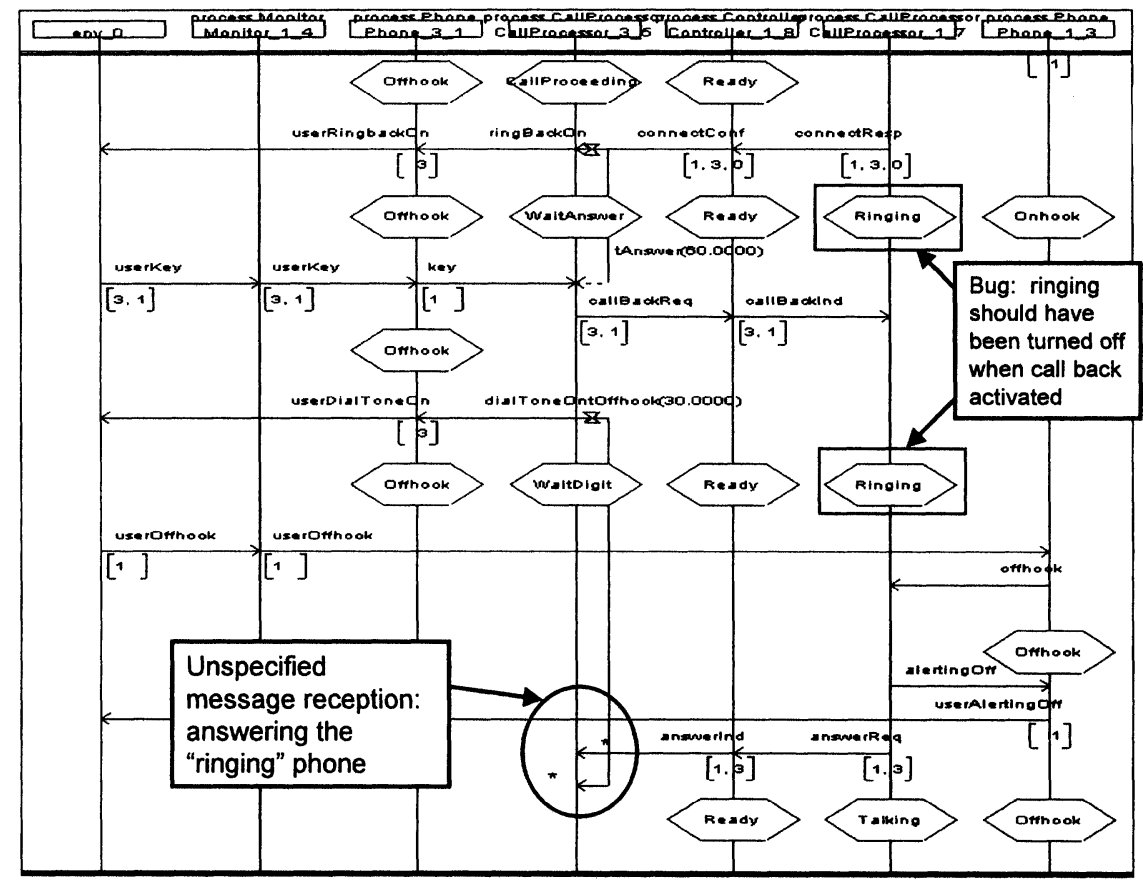

Figure 7. An MSC trace showing an unspecified reception

\section{COVERAGE-BASED TEST GENERATION}

The test cases are constructed using a transition coverage based approach, based on having an SDL transition coverage tool that counted the number of times that a transition was executed during simulation.

We started by creating a test case representing a normal call. We used the coverage tool to display the coverage achieved by this scenario. In our model, a normal call achieves about $30 \%$ transition coverage.

The strategy was then to choose a "target" state and input combination, and attempt to create a TTCN test case that results in covering this particular transition. Figure 8 shows an example of a coverage report produced during this process. After the new TTCN test case had been created, its coverage information would be merged with prior results, to get a new view of the current level of coverage. This process was repeated until all reachable transitions were covered. We found that, on average, we could produce one TTCN test case every fifteen minutes using this approach. 


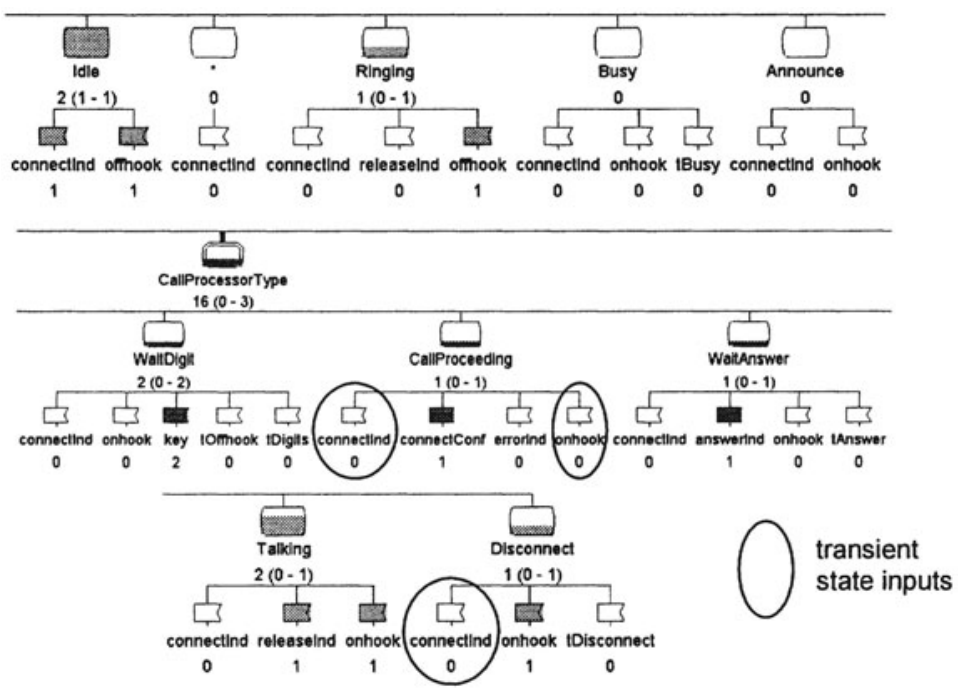

Figure 8. An SDL transition coverage report 


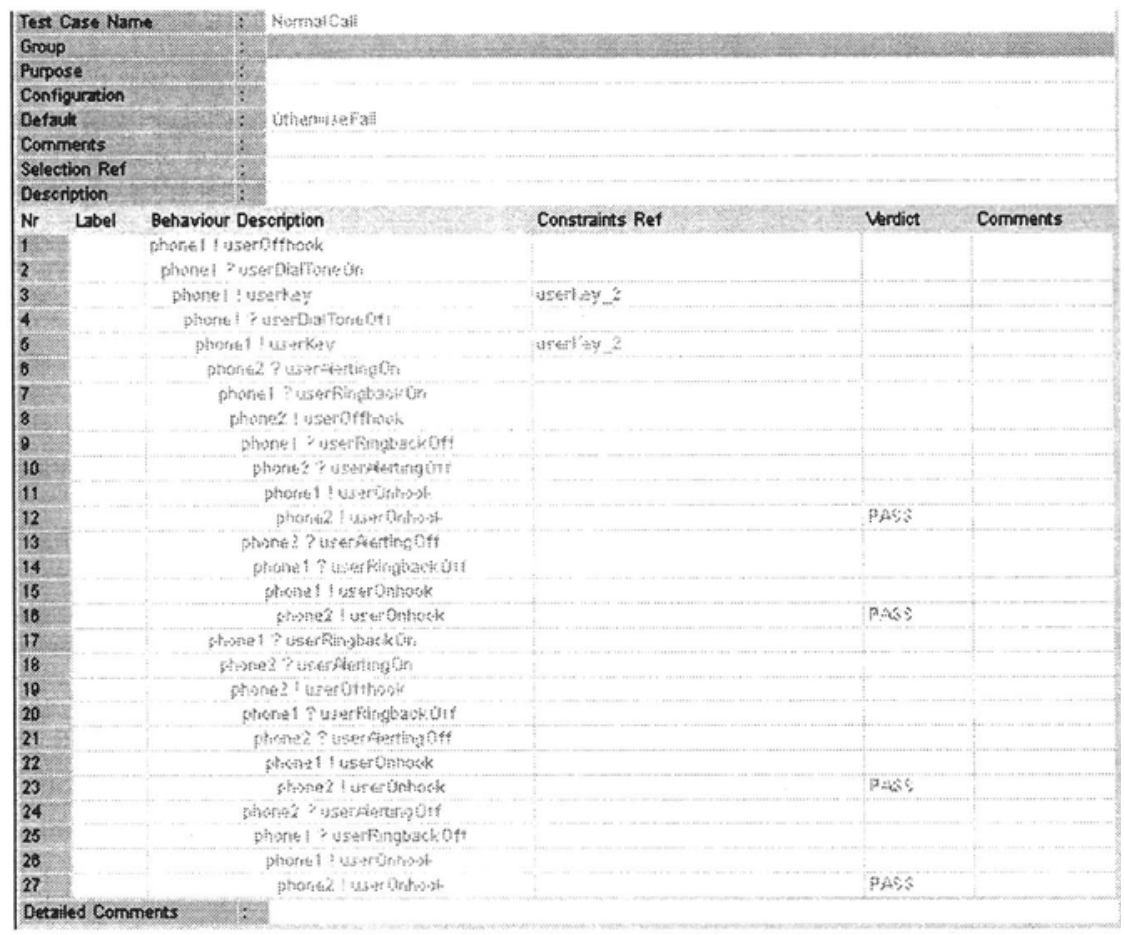

Figure 9. An automatically generated TTCN test case

This approach gave us a set of tests that has the potential for high-yield during execution, because the coverage based approach gave us a series of test cases that concentrates on exceptional cases. Of the eighteen test cases we generated for call processing with no features, there are five cases where timers expire, three cases where the caller aborts before connecting, one call to a non-existent number, and eight cases where incoming calls are rejected with a busy signal.

The coverage-based approach also highlights situations where it may not be possible to create a reliable test case, due to race conditions or transient states. An example of a race condition is when the two phones hang up simultaneously. The disconnect messages may pass in transit, or one or the other might arrive first. An example of a transient state occurs just after when the user hits the final key to dial a number, and before it is determined that the called party exists and is idle. The caller could hang up, or a call could be incoming in this state, even though in real time, the call process is only in this state for a very short length of time.

Creating reliable test cases for an automated execution environment that involves either race conditions or transient states is extremely difficult, if not 
impossible. It requires extremely fine tolerance for timing and control of the test equipment that is used.

\section{PRODUCTIVITY RESULTS}

Before presenting our productivity results, it is necessary to describe how many people were involved and how much product, tool, and methodology knowledge they had. We had one product expert working with two students. The product expert had recently taken courses on the tools and methods, and one of the students learned these while doing the project. The other had prior experience with the use of the tools and methodology, and with telephone switch testing.

During the three months of the project, the students were able to devote most of their time for two months to the project, but during one month, the students were concentrating on other academic work. Figure 10 shows a project time line containing the time taken for various elements of the project $(\mathrm{BC}=$ basic call $)$.

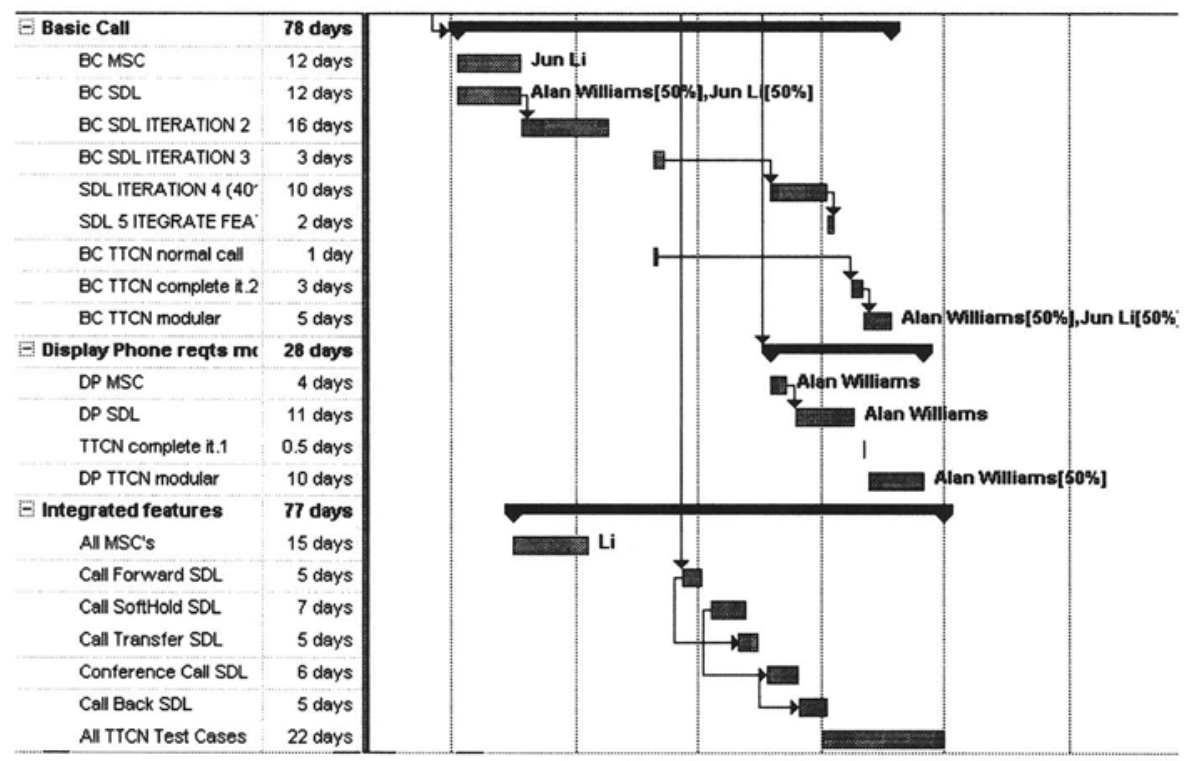

Figure 10. Productivity results 


\section{ISSUES FOR FUNCTIONAL TESTING}

Overall, we were very satisfied with the quality of the test cases that were produced. However, we found two areas where there are opportunities for improvement:

1. ordering of events

2. generation of valid alternatives.

\subsection{Ordering of events}

The sequential nature of an SDL transition imposes an ordering on two consecutive outputs within a single transition. For example, suppose that a single transition has two outputs to the same phone: one to turn on a lamp, and the other to update a screen. When a TTCN test case is generated automatically from this transition, the result is:

? lamp on

? screen update

For user oriented functional testing, the order is irrelevant, because the user is only interested that both events happen within a suitable interval. We do not want to fail the test case if these two events are detected in the opposite order. However, SDL does not provide a mechanism to specify "send two events in no particular order". In fact, forcing the ordering of these two outputs puts unnecessary constraints on the team that implements the specification.

SDL considerations aside, TTCN does allow us to explicitly provide a number of alternative event orderings. But, we found that on the phone set with many display features, there were typically four update messages for each action performed. Because the number of orderings is factorial, there are 24 possible orderings of four events. Specifying each of these every time is not a usable solution.

There needs to be a mechanism in both TTCN and SDL to specify something like the following:

! offhook

\section{? screen | main lamp | line lamp | dial tone}

where the interpretation is that the events separated by vertical bars could happen in any order, but without intervening messages and before subsequent actions. 


\subsection{Generation of valid alternatives}

This point is related to the last one in that the root cause is that we want to leave some message orderings unspecified because a group of them could happen in any order. In this case, we are discussing the process of generating tests, and how to account for such unordered events.

We are using MSCs to guide the test generator, but the MSC contains only a single ordering of possible events (although the co-region feature of MSC-96 allows multiple orderings). Using the example from the previous section, if the MSC happened to have a particular ordering of the messages to a phone's display screen, the two lamps, and the tone generator, the test generator needs to recognize that other alternative orderings are possible. The test generator typically adds a TTCN "otherwise" clause that would cause the test case to fail if any messages other than the one desired arrive at any particular time. But, if there are three other messages that could arrive, and do not represent a violation of desired behaviour, then this needs to be considered by the test generator. The TTCN test case that results should ideally add alternatives that will also lead to a pass verdict.

The tool we were using overcame part of this difficulty by using the state space search mechanism for identifying alternatives. However, instead of taking each alternative branch to its conclusion, if an alternative message arrived, the verdict of the test case was immediately declared to be "inconclusive". The result is that we would still have to generate separate test cases for each possible ordering. But, at least with this approach, valid behaviour would not result in a test case assigning a verdict of fail.

\section{CONCLUSIONS}

Based on our experience with this project, we have developed a number of recommendations:

1. Our approach provides a means of considerably enhancing the productivity of test design engineers. We were able to generate a correct, value-added, TTCN test case every fifteen minutes, which is considerably faster than with a manual approach.

2. Our approach provides a systematic means of improving the quality of designs. Using the state space exploration found a bug in the functionality in a model that had previously undergone testing. More significantly, this bug could have been found long before detailed design, and coding. 
3. The process should be integrated with the design process, so that both designers and testers can take advantage of having an executable model at an early stage.

4. The correct metrics framework for our project only became clear towards the end of the project. We believe that our experience will now allow us to define better metrics that are usable throughout the process.

We would like to further investigate the scalability of our approach, both in terms of the methods, and of the tools used to support them.

\section{ACKNOWLEDGEMENTS}

The authors would like to thank Hasan Ural, Gregor v. Bochmann, Richard Plackowski, Jun Li, and Louise Desrochers for their help with this work, and Daisy Fung, Kelvin Steeden, Bob Armstrong, and Peter Perry for their support..

The authors gratefully acknowledge the support of Mitel Corporation, Nortel Networks, the National Sciences and Engineering Research Council (NSERC), Communications and Information Technology Ontario (CITO), and the University of Ottawa for this research.

\section{REFERENCES}

[1] International Telecommunications Union (ITU-T), Recommendation Z.120: Message Sequence Charts, revised 1996.

[2] International Telecommunications Union (ITU-T), Recommendation Z.100: Specification and Description Language, revised 1996.

[3] International Standards Organization (ISO), OSI Conformance Testing Methodology and Framework - Part 3: The Tree and Tabular Combined Notation, International Standard 9646-3, 1992.

[4] Telelogic Tau tool set, version 3.3, Telelogic AB, Malmo Sweden. 